\title{
Object-based Rician Noise Estimation for MR Images
}

\author{
Pierrick Coupé ${ }^{1}$, José V. Manjon ${ }^{2}$, Elias Gedamu ${ }^{1}$, Douglas Arnold ${ }^{1}$, Montserrat Robles ${ }^{2}$ and D. Louis \\ Collins ${ }^{1}$. \\ 1 McConnell Brain Imaging Centre, Montréal Neurological Institute, McGill University 3801, \\ University Street, Montréal, Canada H3A 2B4. \\ 2 Biomedical Informatics Group (IBIME), ITACA Institute, Polytechnic University of Valencia, \\ Camino de Vera, s/n. 46022 Valencia, Spain.
}

\section{Introduction}

The MR images noise variance is an important measure used for many applications such as denoising, registration or image quality assessment. The real and the imaginary parts of the MR complex raw data are considered as corrupted by white additive Gaussian noises with the same variance [1]. By taking the magnitude of the complex data, the noise is transformed into Rician noise. This noise is usually described by Rayleigh distribution in the background [1] and approximated by Gaussian noise in the foreground when Signal Noise Ratio (SNR) is high enough. These descriptions of noise distribution has been used in the majority of noise estimation methods. Nevertheless, the Rayleigh model of the background can fail when ghosting artefact are presents (i.e. signal different to zeros) [3], and the Gaussian approximation of foreground is no longer valid for low SNR images [3]. To overcome these limitations an object-based estimation taking into account the Rician nature of the noise.

\section{$\underline{\text { Method }}$}

The MAD estimator in wavelet domain [4] is widely used to estimate the variance of Gaussian noise. To obtain an unbiased estimation of $s$ for all the SNR values, we proposed to use the correction procedure introduced in [2]. This analytical correction is based on an iterative estimation of the SNR in presence of Rician noise (see Figure 1).

First, the object is extracted in the wavelet domain by k-means segmentation in the lowest subband. After, the MAD estimator is computed on wavelet coefficients corresponding to the object in the highest subband. Then, this first estimation $\mathrm{s}^{\wedge}$ is iteratively corrected with equation given in Figure 1. Finally, the estimation $\mathrm{sn}^{\wedge}$ of the Rician noise variance is obtained.

\section{$\underline{\text { Result }}$}

Synthetic Data: To evaluate our method, synthetic T1-weighted MR data of Brainweb database [5] was corrupted with different levels of Rician noise (2 to $15 \%$ ). Ghosting artefacts and inhomogeneity have been also added (see Fig 2). To estimate the accuracy of the method, the ratio between the estimated standard deviation and the applied standard deviation is computed for all the levels of noise. Our method was compared with the Maximum likelihood (ML) background method described in [3] and the original MAD estimator. The results are given in Figure 3. 
T1-w data: Experiment on real data were performed on 23 T1-w MR images of 256x256x56 voxels acquired with the same sequence and device (1.5T Genesis Signa GE Medical system). The bronze standard is computed as the mean level of noise over all the data. The level of noise for a real data is obtain by extracting an artifact-free region in the background [6]. The results are given in Figure 4.

\section{Conclusion}

The results on the BrainWeb phantom show that the proposed object-based method is more robust than background-based method. Moreover, the proposed Rician adaptation of the MAD produces better results than classical MAD estimator, especially for low SNR. Finally, experiments on real data show that the proposed method accurately estimate the variance of noise.

\section{$\underline{\text { References }}$}

[1] H. Gudbjartsson and S. Patz. The Rician distribution of noisy MRI data. Magnetic Resonance in Medicine, 34:910-914, 1995.

[2] C. G. Koay and P. J. Basser. Analytically exact correction scheme for signal extraction from noisy magnitude MR signals. Journal of Magnetic Resonance, 179(2):317-322, April 2006.

[3] J. Sijbers, D. Poot, A. J. den Dekker, and W. Pintjens. Automatic estimation of the noise variance from the histogram of a magnetic resonance image. Physics in Medicine and Biology, 52(5):1335-1348, 2007.

[4] D.L. Donoho and I.M. Johnstone. Ideal spatial adaptation by wavelet shrinkage. Biometrika, 81(3):425-455, 1994.

[5] D.L. Collins, A.P. Zijdenbos, V. Kollokian, J.G. Sled, N.J. Kabani, C.J. Holmes, and A.C. Evans. Design and construction of a realistic digital brain phantom. IEEE Transactions on Medical Imaging, 17(3):463-468, 1998.

[6] E. L. Gedamu, D. L. Collins, and D. L. Arnold. Automated quality control of brain MR images. Journal of magnétique Resonance Imaging, 28(2):308?319, August 2008.

$$
\hat{\sigma}_{n}=\sqrt{\hat{\sigma}^{2} / \xi(\theta)}
$$

where $\theta$ is the SNR value and $\xi$ the correction factor expressed as:

$$
\xi(\theta)=2+\theta^{2}-\frac{\pi}{8} \times \exp \left(-\frac{\theta^{2}}{2}\right)\left(\left(2+\theta^{2}\right) I_{0}\left(\frac{\theta^{2}}{4}\right)+\theta^{2} I_{1}\left(\frac{\theta^{2}}{4}\right)\right)^{2}
$$

where $I_{1}$ is the first order modified Bessel function.

Figure 1. Equation of the iterative estimation of Rician noise variance. 

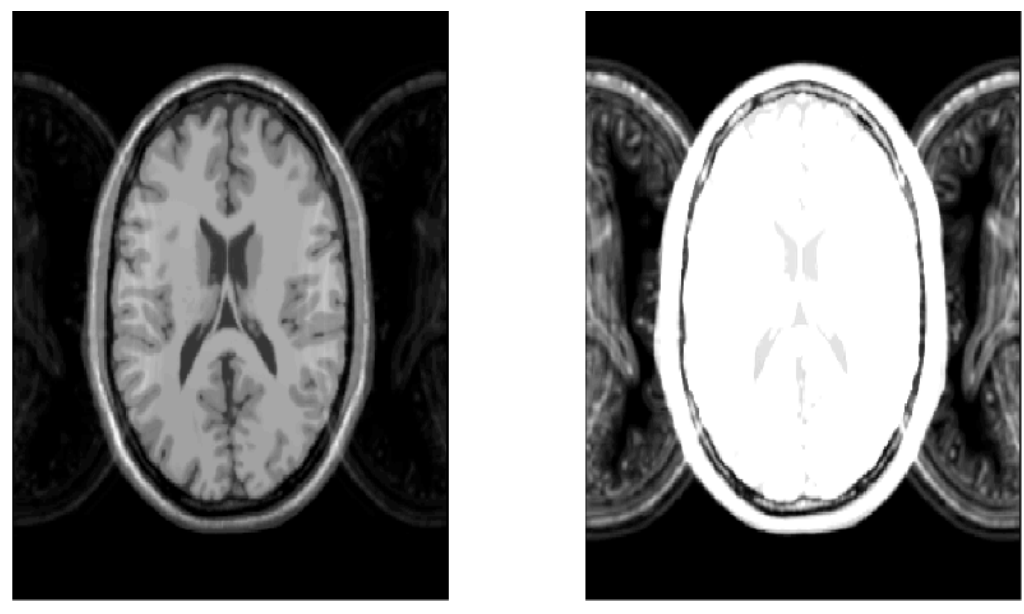

Figure 2. Simulated ghosting artefacts on Brainweb with $20 \%$ of inhomogeneity.

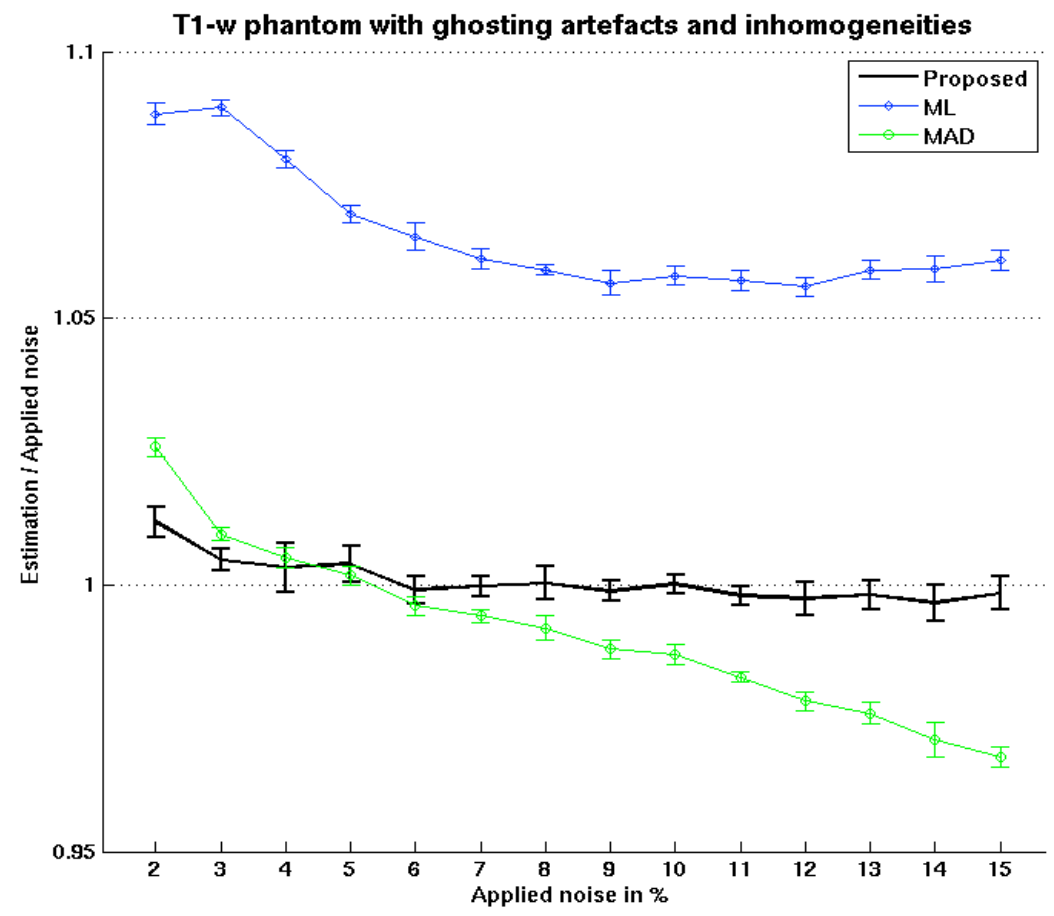


Figure 3. Results obtained by the compared methods for all the level of noise on synthetic image with ghosting artefacts and $20 \%$ of inhomogeneities.

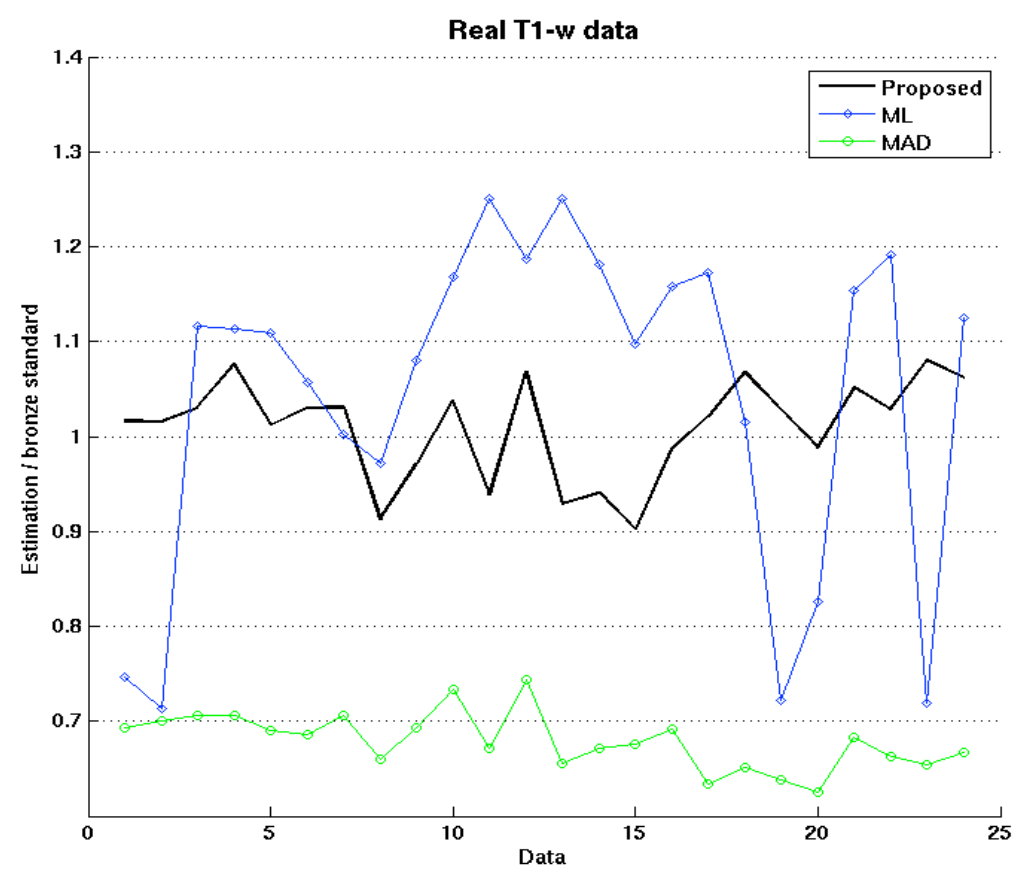

Figure 4. Results obtained by the compared methods for all the real data. 